\title{
Cirugía pulmonar en tuberculosis
}

\author{
Macarena Rodríguez ${ }^{1}$, José Manuel Munita ${ }^{1}$, Daniel Péreza, \\ Felipe Bannura2 ${ }^{2}$ Juan Carlos Rodríguez ${ }^{3}$, Patricio Rodríguez ${ }^{2}$. \\ Surgery for pulmonary tuberculosis. \\ Review of 33 operated patients
}

Background: Surgical treatment for pulmonary tuberculosis is mainly limited to the management of sequelae such as bronchiectasis, hemoptysis and brochopleural fistulae. Aim: To review the data of patients who underwent surgical treatment for pulmonary tuberculosis. Material and methods: Retrospective review of 33 patients aged 18 to 73 years (24 males) who underwent lung resection surgery for the management of pulmonary tuberculosis. Follow-up data were obtained from outpatient visit records and registries of the national tuberculosis program. Results: The reasons to perform surgery were the following: fifteen for hemoptysis, nine for lung destruction and nine for an active and multiresistant disease. No patient died in the postoperative period. The morbidity observed included empyema $(n=5)$, pneumothorax $(n=2)$, bronchopleural fistula $(n=2)$ and hemothorax $(\mathrm{n}=2)$. At six months of follow up, six of the nine patients with active tuberculosis had negative acid-fast bacilli on sputum smear. Two of these patients died, one due to respiratory failure and another by an unrelated cause. Both dead patients had negative acidfast bacilli on sputum smear. Conclusions: Surgery in pulmonary tuberculosis has a high rate of complications but may be useful in selected patients (Rev Méd Chile 2009; 137: 234-9).

(Key words: Hemoptysis; Thoracic surgery; Tuberculosis, pulmonary)

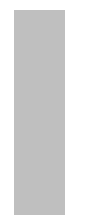

\footnotetext{
Recibido el 11 de junio, 2008. Aceptado el 27 de octubre, 2008.

Sin apoyo financiero.

${ }^{1}$ Servicio de Medicina, Hospital Padre Hurtado, Facultad de Medicina, Clínica Alemana-Universidad del Desarrollo. ${ }^{2}$ Servicio de Cirugía y ${ }^{3}$ Servicio de Medicina, Hospital INER y CT.

anterno de Medicina de la Facultad de Medicina, Clínica Alemana-Universidad del Desarrollo
}

$\mathrm{E}$ xisten alrededor de 9 millones de casos nuevos de tuberculosis (TBC) y 2 millones de muertes anuales en el mundo ${ }^{1}$. La TBC es una pandemia cuya incidencia global está creciendo a pesar de intensos esfuerzos por controlarla. En países desarrollados, las cifras de TBC han dismi-

Correspondencia a: Dr. José Manuel Munita Sepúlveda. Esperanza 2150, paradero 28 Santa Rosa, San Ramón. Teléfonos: 8131027-5760711-5760710. Santiago Chile.

E mail: munita.jm@gmail.com nuido dramáticamente, pero lograr la erradicación de la enfermedad no ha sido posible en ninguna parte del mundo.

En nuestro país hemos alcanzado el "umbral de eliminación" de la enfermedad, con una incidencia de 16/100.000 habitantes, sin embargo existe una gran variabilidad entre las distintas regiones, con cifras tan altas como 38,2/100.000 habitantes en la primera región y otras como 3,4/ 100.000 habitantes en la zona correspondiente al Servicio de Salud Aconcagua ${ }^{2}$.

Los principales obstáculos hacia la eliminación de la TBC son el desarrollo de multirresistencia -definido 
como resistencia simultánea a la rifampicina e isoniacida-, la ausencia de programas de control adecuados, las migraciones, la pobreza y la coinfección con $\mathrm{VIH} / \mathrm{SIDA}$.

Existen 2 tipos de resistencias, la primaria, que se presenta en pacientes vírgenes al tratamiento y la secundaria o adquirida, propia de pacientes ya tratados. En nuestro país la resistencia secundaria es bastante mayor que la primaria: $3,8 \%$ y $0,7 \%$, respectivamente. Las cifras de multirresistencia han aumentado en los últimos años con importantes implicancias económicas y pronósticas, pues la terapia es exitosa en no más de 50\% de los casos. En el caso de la polirresistencia -i.e. resistencia a otras drogas además de rifampicina e isoniacidael éxito terapéutico es aún menor y en ocasiones no existen esquemas curativos. Esto plantea la necesidad de alternativas de rescate como la resección del órgano comprometido.

El manejo quirúrgico de la TBC pulmonar surgió en el siglo XIX y fue por muchos años la única alternativa terapéutica. El desarrollo de la terapia antibiótica asociada permitió que la enfermedad se pudiera controlar farmacológicamente y disminuyó las indicaciones quirúrgicas, limitándolas básicamente al manejo de las secuelas ${ }^{3}$, entre las que se encuentran las bronquiectasias, la hemoptisis masiva o recurrente (en ocasiones asociada a colonización por aspergillus), la fístula broncopleural y, por último, la destrucción pulmonar, que conlleva complicaciones como reactivación, aspergiloma y hemoptisis.

La emergencia de TBC multirresistente ha hecho resurgir el interés por las terapias quirúrgicas. Hoy se usa no sólo para el manejo de lesiones secuelares, sino también para casos muy seleccionados de TBC activa, entre los que se incluyen: la resistencia a más de cuatro drogas, múltiples recaídas en tratamiento y persistencia de baciloscopias positivas a los 4-6 meses a pesar de terapia apropiada $^{4}$.

Existen series extranjeras de cirugía en TBC resistente activa que obtienen tasas de curación entre 93\%-94\%5,6. Cabe señalar que la cirugía es sólo una terapia adyuvante y debe utilizarse siempre en asociación con antibióticos.

Llama la atención que la cirugía de resección pulmonar aún forme parte del arsenal terapéutico, siendo la TBC una enfermedad infecciosa para la cual existe un tratamiento antibiótico eficaz. En nuestro país no existen series publicadas en relación al tratamiento quirúrgico de la TBC. El objetivo de este trabajo es describir el tipo de pacientes que están recibiendo tratamiento quirúrgico: la proporción de pacientes con TBC activa, el motivo de la indicación y el tipo de resistencia que presentan. En los pacientes con secuelas, describir cuáles son las lesiones y la temporalidad de ellas en relación al diagnóstico.

\section{Material y MÉTOdO}

El estudio incluye los pacientes con TBC que fueron sometidos a cirugía torácica desde enero del año 2000 hasta julio del año 2006 en el Instituto Nacional de Enfermedades Respiratorias y Cirugía Torácica, centro de referencia nacional.

Los casos se obtuvieron a partir del registro de pabellón. Se realizó una evaluación retrospectiva de las fichas clínicas por 2 revisores independientes registrándose los datos en un formulario estándar. En aquellos casos en que hubo diferencias en el registro se recurrió a una tercera revisión. Las cirugías incluidas son sólo aquellas con fines terapéuticos.

Algunos pacientes tenían lesiones secuelares y otros lesiones activas. Los criterios para considerar las lesiones como secuelares fueron: historia previa de tuberculosis, lesiones pulmonares compatibles y ausencia de BAAR en el tejido pulmonar resecado, tanto en el examen directo como en el cultivo. El criterio diagnóstico para tuberculosis activa fue bacteriológico. Se consideró colonización por Aspergillus la presencia de una imagen pulmonar sugerente asociada a serología positiva o la presencia de este hongo en el estudio anatomopatológico.

El motivo que se señala como determinante de la indicación quirúrgica es aquél definido por el equipo tratante.

El estudio preoperatorio incluyó una radiografía de tórax, escáner de tórax, estudios de función pulmonar y cintigrama de perfusión (para predecir la función respiratoria postoperatoria). Esto no se realizó en los casos de hemoptisis masiva que fueron sometidos a cirugía de urgencia. En relación a las complicaciones, se hace la distinción entre fuga aérea prolongada que se define como la fuga aérea por el tubo pleural por más de cinco 
días y la fístula broncopleural que consiste en la fuga de aire a través del muñón bronquial.

El seguimiento se obtuvo de los registros de controles postoperatorios y del programa nacional de tuberculosis del Ministerio de Salud. En el caso de los pacientes fallecidos la causa de muerte es aquella que consta en el certificado de defunción de acuerdo al Registro Civil.

\section{Resultados}

Un total de 33 pacientes fueron sometidos a cirugía de resección pulmonar. La edad promedio fue de 45 años (rango 18 a 73) e incluyó 24 hombres y 9 mujeres. Todos los pacientes eran de nacionalidad chilena a excepción de un peruano. Seis pacientes tenían antecedentes de cirugía pulmonar: 4 con lobectomía previa, 1 con cavernotomía y otro había sido sometido a pleurodesis por neumotórax recurrente.

La comorbilidad se registra en la Tabla 1. Hubo 15 pacientes sin patología asociada y sólo cinco pacientes con más de una comorbilidad.

La indicación quirúrgica más frecuente fue la hemoptisis (15 pacientes), siendo la mayor parte de ellas masivas. Otras indicaciones fueron pulmón destruido (9 pacientes) y TBC resistente activa ( 9 pacientes). Uno de los pacientes operados por TBC activa presentaba además hemoptisis, sin embargo, ésta no fue el motivo de la cirugía. Estas frecuencias están expresadas en la Tabla 2.

Cabe destacar que los 9 casos de TBC activa eran no multi sino polirresistentes. Todos presentaban resistencia secundaria y en 5 de ellos se

\section{Tabla 1. C omorbilidad}

\begin{tabular}{|lr|}
\hline Comorbilidad & Número \\
\hline Diabetes mellitus & 2 \\
HTA & 4 \\
Tabaquismo & 15 \\
Alcoholismo & 1 \\
Drogadicción & 1 \\
HIV & 1 \\
Retardo mental & 1 \\
\hline
\end{tabular}

consignaron problemas de adherencia al tratamiento. Los factores asociados con mala adherencia fueron alcoholismo, intolerancia a las drogas y abandono precoz de la terapia. En relación a la disponibilidad de drogas, en dos ocasiones se consigna que la cicloserina no fue entregada en forma oportuna, argumentándose ausencia de la droga en nuestro país. De los nueve pacientes operados por TBC resistente activa 7 recibieron, en promedio, 2 meses de terapia antituberculosa adecuada (según los perfiles de resistencia), sin lograr negativizar las baciloscopias. El único paciente que fue operado con baciloscopias negativas recibió 11 meses de tratamiento preoperatorio, un paciente fue operado por hemoptisis masiva sin recibir terapia previa. Los pacientes operados con TBC resistente activa se detallan en la Tabla 3.

Del total de pacientes 11 tenían evidencias de aspergilosis pulmonar, en 7 de ellos la hemoptisis fue la indicación quirúrgica y sólo uno de éstos presentaba además TBC activa, siendo el único paciente VIH positivo.

Los pacientes operados por secuelas de TBC presentaban, en promedio, 18 años desde el diagnóstico de TBC, con un rango de 1 a 56 años.

El tipo de cirugías realizadas se enumera en la Tabla 4 .

Once pacientes presentaron complicaciones (Tabla 5), dos de los cuales desarrollaron más de una: un caso de hemotórax que requirió reintervención y evolucionó con un empiema y otro caso de una fístula broncopleural y empiema. Sólo una de las complicaciones se presentó en un paciente con TBC activa y no hubo mortalidad operatoria ni postoperatoria precoz.

En relación al seguimiento realizado hasta mayo de 2007, existe un paciente operado por TBC activa, cuya situación vital desconocemos. De aquellos pacientes operados por secuelas de TBC dos fallecieron; uno por EPOC descompensado y

\section{Tabla 2. Indicación quirúrgica}

\begin{tabular}{|lc|}
\hline Indicación quirúrgica & Número \\
\hline Hemoptisis masiva & 9 \\
Hemoptisis recurrente & 6 \\
Pulmón destruido & 9 \\
TBC resistente & 9 \\
\hline
\end{tabular}


Tabla 3. Pacientes con T BC resistente activa

\begin{tabular}{|cccccc|}
\hline Pacientes & Edad & Sexo & Comorbilidad & Procedimiento & Complicaciones \\
\hline 1 & 48 & Masculino & Tabaquismo/HTA & Neumonectomía & No \\
$2^{*}$ & 32 & Masculino & Tabaquismo & Neumonectomía & Hemotórax \\
3 & 63 & Masculino & No & Lobectomía & No \\
4 & 35 & Masculino & No & Bilobectomía & No \\
5 & 38 & Masculino & No & Bilobectomía + resección segm. & No \\
6 & 33 & Femenino & No & Neumonectomía & No \\
7 & 27 & Femenino & No & Lobectomía & No \\
8 & 18 & Masculino & No & Nobectomía & No \\
9 & 35 & Masculino & Tabaco/VIH $(+)$ & & \\
\hline
\end{tabular}

*Este paciente presentaba hemoptisis además de tuberculosis activa resistente

Tabla 4. Tipo de cirugías realizadas

\begin{tabular}{|lr|}
\hline Cirugía & Número \\
\hline Lobectomía & 18 \\
Bilobectomía & 2 \\
Bilobectomía y resección segmentaria & 1 \\
Neumonectomía & 12 \\
\hline
\end{tabular}

Tabla 5. Tabla de complicaciones

\begin{tabular}{|lc|}
\hline Complicaciones & Número \\
\hline Empiema & 5 \\
Fuga aérea prolongada & 1 \\
Neumotórax & 2 \\
Fístula broncopleural & 2 \\
Hemotórax & 2 \\
Absceso pared & 1 \\
Total de pacientes* & 11 \\
\hline
\end{tabular}

*2 pacientes presentaron más de una complicación

otro por cáncer pulmonar. En el grupo de pacientes con TBC resistente activa hay dos fallecidos, siendo las causas de defunción una insuficiencia respiratoria aguda y un accidente de tránsito, ambos seis meses después de la cirugía y aun recibiendo tratamiento antituberculoso. En relación a los otros seis pacientes con TBC resistente,
4 presentaron curación confirmada bacteriológicamente y 2 abandonaron la terapia; uno a los 12 y el otro a los 10 meses de tratamiento, ambos con baciloscopias negativas en esa fecha.

\section{Discusión}

La cirugía torácica en enfermedades inflamatorias benignas se considera un procedimiento de muy alto riesgo, que debe evitarse siempre que sea posible. A pesar de esto, existen situaciones en las cuales ésta es la única alternativa terapéutica.

Los casos de TBC polirresitente son habitualmente derivados a este Instituto, puesto que es el centro de referencia nacional. Esto nos permite suponer que nuestra serie es un buen reflejo de las cirugías pulmonares realizadas en pacientes con TBC activa en nuestro país, por el contrario, la cirugía en lesiones pulmonares inactivas podría realizarse en distintos centros que dispongan de cirugía torácica.

La mortalidad operatoria reportada en las distintas series fluctúa entre $0 \%$ y $3,3 \%{ }^{4,7,8}$ siendo mayor en los casos de tuberculosis activa. En nuestra serie no hubo mortalidad operatoria precoz ni tardía (a 30 días), lo que puede explicarse por la adecuada evaluación y selección de los pacientes, además de la experiencia del equipo quirúrgico.

La morbilidad de esta cirugía es elevada, presentándose en 33\% en nuestro grupo; las cifras 
reportadas en la literatura varían entre 15\% y $40 \% 3,9,10$. Cinco pacientes evolucionaron con empiema pleural y dos con fístula broncopleural, una de las complicaciones más temidas. Ninguna de estas complicaciones se observó en pacientes con TBC activa, a diferencia de lo descrito en otras series.

En relación a los pacientes con TBC activa multirresistente, existe controversia en la literatura en relación al tiempo óptimo de terapia farmacológica previo a la cirugía. Inicialmente se sugerían al menos tres meses de tratamiento, considerando el tiempo promedio de negativización de las baciloscopias $^{10}$. El aumento global de la resistencia ha motivado una conducta cada vez más agresiva, considerándose la cirugía en forma precoz $^{11}$. No existen trabajos comparativos randomizados al respecto y, a la fecha, el momento quirúrgico está determinado exclusivamente por el juicio clínico.

De ocho pacientes con TBC resistente sólo uno falleció por esta causa. Aun si los pacientes que se perdieron del seguimiento presentaran TBC activa, el pronóstico de la cirugía sería mejor que el pronóstico reportado sin ella. Lamentablemente no tenemos el seguimiento completo, esto refleja lo difícil que resulta el control de esta patología, aun en países como el nuestro donde la terapia es supervisada y existe un adecuado registro y seguimiento de los enfermos.

La falta de adherencia a la terapia es el mayor determinante de la presencia de resistencia farmacológica. En este sentido, es discutible que pacientes con TBC multirresistente fuesen autorizados por sus respectivos centros de salud para autoadministrarse la terapia. Dada la relevancia del problema de la multirresistencia, no sólo en términos epidemiológicos sino también económicos, la terapia supervisada no debiera suspenderse nunca en un paciente con multirresistencia.

De los pacientes con lesiones secuelares, la mayoría fueron operados muchos años después de la TBC activa, lo que señala que muchos de los pacientes que operamos hoy fueron diagnosticados y tratados en la época de los inicios del DOTS (del inglés directly observed therapy-short course) en nuestro país, cuando aún no tenía la cobertura adecuada. Por otra parte existen casos que son operados por pulmón destruido a sólo un año de finalizado el tratamiento, es decir con lesiones extensas, lo que sugiere que éste fue iniciado en forma tardía. Esto nos alerta a no descuidar la importancia de la pesquisa precoz.

La hemoptisis es una condición que genera riesgo vital y, en presencia de lesiones residuales, tiende a recurrir de no establecerse una terapia adecuada. En los pacientes sometidos a terapia médica, se han descrito cifras de 36\% de recurrencia, con una mortalidad de $45 \%{ }^{12}$. De nuestros pacientes, 9 fueron operados con el diagnóstico de hemoptisis masiva, sólo uno de ellos ha fallecido a la fecha. En este tipo de enfermos la hemoptisis, cuando es recurrente o masiva, requiere de cirugía, ya que es el único tratamiento definitivo en nuestro medio. En otros lugares la embolización puede ser una alternativa, sin embargo este no es un recurso disponible en el sistema público de salud y se utiliza excepcionalmente, cuando la función pulmonar no permite la cirugía.

Si bien el Aspergillus se encuentra con frecuencia en cavidades tuberculosas ya curadas, la infección por Aspergillus de una cavidad infectada por Mycobacterium tuberculosis es rarísima ${ }^{13}$. Esto se observó en un solo caso, siendo el único paciente con VIH/SIDA. Lo anterior sugiere que probablemente se necesita un marcado deterioro de la inmunidad para que exista coinfección. La parasitación de las cavidades por Aspergillus puede ocasionar dos cuadros diferentes: el aspergiloma simple o la forma localmente invasora en la que hay una reacción fibrosante en las zonas pericavitarias. En nuestro medio el aspergiloma es una de las causas más frecuentes de hemoptisis y si bien no hay series prospectivas, lo esperable es que el sangrado recurra, por lo que la cirugía es una opción que debe considerarse.

La cirugía en pacientes con lesiones pulmonares por TBC, ya sea secuelares o activas, ha demostrado ser un herramienta útil a pesar de su alta morbilidad. Considerando el aumento mundial de la tuberculosis resistente, la cirugía probablemente jugará un papel cada vez mayor. Se desconoce el momento oportuno de esta terapia, tema que pudiera ser objeto de futuras investigaciones. 


\section{REFERENCIAS}

1. WHO REPORT 2008. Global tuberculosis control, surveillance, planning, financing. Pág 11. Disponible en www.who.int. (Consultado el 25 de mayo de 2008).

2. ZuÑiga M. Tuberculosis en Chile: ¿Es posible la eliminación? Cuad Méd Soc (Chile) 2004, 44: 144-61.

3. Takeda S, Maeda H, Hayakawa M, Sawabata N, Maekura R. Current surgical intervention for Pulmonary Tuberculosis. Ann Thorac Surg 2005; 79: 959-63.

4. Van Leuven M, De Groot M, Shean K, Von Oppell UO. Pulmonary resection as an adjunct in the treatment of multiple drug-resistant tuberculosis. Ann Thorac Surg 1997; 63: 1368-73.

5. Kir A, Inci I, Torun T, Atasalihi A, Tahaoglu K. Adjuvant resectional surgery improves cure rates in MDR tuberculosis. J Thorac Cardiovasc Surg 2006; 131: 693-6.

6. Shiraishi Y, Nakajima Y, Katsuragi N, Kurai M, TakahasHI N. Resectional surgery combined with chemotherapy remains the treatment of choice for multidrug resistant tuberculosis. J Thorac Cardiovasc Surg 2004; 128: 523-8.

7. Pomerantz BJ, Cleveland Jc Jr, Olson Hk, Pomerantz M. Pulmonary resection for multi-drug resistant tuber- culosis. J Thorac Cardiovasc Surg 2001; 121: 448-53.

8. Shiraishi Y, Nakajima Y, Katsuragi N, Kurai M, TakahasHI N. Resectional surgery combined with chemotherapy remains the treatment of choice for multidrug-resistant tuberculosis. J Thorac Cardiovasc Surg 2004; 128: 523-8.

9. Reed CE, Parker EF, Crawford FA JR. Surgical resection for complications of pulmonary tuberculosis. Ann Thorac Surg 1989; 48: 165-7.

10. Pomerantz M, Madsen L, Goble M, Iseman M. Surgical management of resistant mycobacterial tuberculosis and other mycobacterial pulmonary infections. Ann Thorac Surg 1991; 52: 1108-12.

11. Lalloo Ug, Naidoo R, Ambaram A. Recent advances in the medical and surgical treatment of multi-drug resistant tuberculosis. Curr Opin Pulm Med 2006; 12: 179-85. Review.

12. Knott-Craig CJ, Oostuizen JG, Rossouw G, Joubert JR, BARNARD PM. Management and prognosis of massive hemoptysis. Recent experience with 120 patients. J Thorac Cardiovasc Surg 1993; 105: 394-7.

13. Kohno S, Hatano T, Yamada H, Maesaki S, Yasuoka A, KaKu M, Koga H, Hara K. Superinfection of chronic necrotizing pulmonary aspergillosis by Mycobacterium tuberculosis. Intern Med 1992; 31: 540-3. 